\title{
Multiple Cold Tolerance Trait Phenotyping Reveals Shared Quantitative Trait Loci in Oryza sativa
}

\author{
Naoki Shimoyama, Melineeh Johnson, André Beaumont and Michael Schläppi i (D)
}

\begin{abstract}
Background: Developing chilling tolerant accessions of domesticated Asian rice is a potential source of significant crop improvement. The uniquely chilling sensitive nature of the tropically originating Oryza sativa make it the most important cereal crop that can gain significantly from improved tolerance to low temperatures. However, mechanisms underlying this complex trait are not fully understood. Oryza sativa has two subspecies with different levels of chilling tolerance, JAPONICA and INDICA, providing an ideal tool to investigate mechanistic differences in the chilling stress tolerance responses within this important crop species.

Results: The Rice Diversity Panel 1 (RDP1) was used to investigate a core set of Oryza sativa accessions. The tools available for this panel allowed for a comprehensive analysis of two chilling tolerance traits at multiple temperatures across a 354-cultivar subset of the RDP1. Chilling tolerance trait values were distributed as mostly subpopulation specific clusters of Tolerant, Intermediate, and Sensitive accessions. Genome-wide association study (GWAS) mapping approaches using all 354 accessions yielded a total of 245 quantitative trait loci (QTL), containing 178 unique QTL covering 25\% of the rice genome, while 40 QTL were identified by multiple traits. QTL mappings using subsets of rice accession clusters yielded another $255 \mathrm{QTL}$, for a total of $500 \mathrm{QTL}$. The genes within these multiple trait QTL were analyzed for Gene Ontology (GO) term and potential pathway enrichments. Terms related to "carbohydrate biosynthesis", "carbohydrate transmembrane transport", "small molecule protein modification", and "plasma membrane" were enriched from this list. Filtering was done to identify more likely candidate pathways involved in conferring chilling tolerance, resulting in enrichment of terms related to "Golgi apparatus", "stress response", "transmembrane transport", and "signal transduction".

Conclusions: Taken together, these GO term clusters revealed a likely involvement of Golgi-mediated subcellular and extracellular vesicle and intracellular carbohydrate transport as a general cold stress tolerance response mechanism to achieve cell and metabolic homeostasis under chilling stress.
\end{abstract}

Keywords: Abiotic stress, Chilling stress, Genome wide association study (GWAS), Golgi, Rice (Oryza sativa L.), Secretory pathway, Ubiquitination

\footnotetext{
* Correspondence: michael.schlappi@marquette.edu

Department of Biological Sciences, Marquette University, Milwaukee, WI 53233, USA
} 


\section{Background}

\section{Rising Global Food Demand Requires Significant} Improvement in Abiotic Stress Tolerance

It was recently estimated that, by the year 2050, a $50 \%$ increase in food production is required to meet the needs of a growing global population (Food and Agriculture Organization of the United Nations (FAO) 2017). However, the rates of increase for major crops are falling significantly short to meet this demand. One possible method for increasing crop yields is to reduce losses caused by abiotic stress. Significant yield losses occur due to chilling stress every year (da Cruz et al. 2013; Singh et al. 2017). As global climate change continues to affect the predictability of seasonal temperatures, it will be important to significantly improve the resilience of our crops to aberrant cold snaps and unseasonably cold temperatures during critical stages of plant development, such as the young seedling stage.

\section{Rice as a Natural Model for Understanding Chilling Stress Tolerance Response Mechanisms}

Plant chilling tolerance is a complex trait and its mechanisms have not yet been fully elucidated. Understanding cold stress tolerance response mechanisms is paramount to improve chilling tolerance of crop plants such as rice. Domesticated rice, Oryza sativa L., is a major food source for about half of the world population (McLean et al. 2013). However, current annual increases in rice production are not sufficient to meet the needs of our growing population. Unlike other staple crops, rice is uniquely susceptible to chilling stress due to its tropical and subtropical origins. Every year, chilling stress causes major yield loss in the rice crop (da Cruz et al. 2013). To eventually reduce such losses, our study aims to better understand cold stress tolerance response mechanisms in young seedlings, a critical stage for rice phenology.

We use domesticated rice as a model to investigate plant chilling tolerance (Schläppi et al. 2017; Liu et al. 2018; Shi et al. 2020). Rice is a useful tool for studying chilling tolerance due to its significant chilling sensitivity because of the tropical origins of its ancestors. The threshold temperature for chilling stress induced damage in rice is around $10^{\circ} \mathrm{C}$ while other major crops such as winter wheat can withstand freezing temperatures (da Cruz et al. 2013). This feature indicates that compared to other major crops, improving chilling tolerance in domesticated rice may have one of the largest impacts on increasing crop yields. Additionally, due to artificial human selection, there are two distinct varietal groups within the rice species with significantly different chilling tolerance abilities (Shakiba et al. 2017; Liu et al. 2018). The JAPONICA subspecies, consisting of temperate japonica, tropical japon$i c a$, and aromatic subpopulations, is derived from varieties that were artificially selected and cultivated in temperate regions such as northern China, Japan, and Korea, where resistance to chilling temperatures was necessary. The INDICA subspecies, consisting of aus and various indica subpopulations, is derived from varieties that were artificially selected and cultivated in tropical and subtropical regions of southern and south eastern Asia, where selection for chilling tolerance traits was not necessary. These divergent varietal groups are extremely useful in identifying potential pathways associated with chilling tolerance. The fact that chilling tolerance within members of the $J A$ PONICA subspecies was developed through artificial human selection instead of targeted genetic engineering raises the possibility that different chilling stress tolerance response mechanisms were selected in different rice accessions or entire subpopulations. Similarly, the large diversity found in the INDICA subspecies might provide insight into which chilling tolerance pathways were selected against in favor of other pathways that optimized other agricultural parameters.

\section{Genome-Wide Association Study (GWAS) Mapping of Chilling Tolerance Traits}

Studying a wide range of genetically diverse population of rice cultivars provides us with a species-wide insight into chilling tolerance. By uncovering the mechanisms underlying the varietal group differences in chilling tolerance, it may be possible to improve chilling tolerance with limited genetic interventions by utilizing resistance systems already employed. To this end, the Rice Diversity Panel 1 (RDP1) developed as part of the Rice Diversity Project is a useful tool. It is a panel of 424 purified, homozygous accessions that are representative of the entire $O$. sativa species containing the five major subpopulations (aromatic, aus, indica, temperate japonica, and tropical japonica) and their admixed accessions (Eizenga et al. 2014; McCouch et al. 2016). This panel is additionally useful due to the genomewide association study (GWAS) mapping pipeline developed by McCouch et al. 2016, which allows for in-house GWAS mapping even with limited computational resources. For these reasons, a subset of RDP1 accessions was used for phenotyping and GWAS mapping.

\section{Purpose of this Study}

The major aim of this study was to help elucidating chilling stress tolerance response mechanisms in the generally cold sensitive crop species Oryza sativa. We show via genotype-based principal component analyses (PCA) combined with cold tolerance trait phenotyping analyses done at different temperatures that the RDP1 contains three distinct, mostly subpopulation-specific, clusters of accessions classified as chilling tolerant (Tolerant), intermediate chilling tolerant (Intermediate), and chilling sensitive (Sensitive). Through GWAS mapping using all accessions or accessions of the Tolerant and 
Sensitive clusters and accessions within clusters, we identified 500 QTL and a large number of QTL regionassociated chilling tolerance candidate genes. We then used several filtering methods to reduce the number of candidate genes to identify the most probable chilling tolerance response pathways that are significantly altered between the chilling tolerant JAPONICA (aromatic; temperate japonica; tropical japonica) and chilling sensitive INDICA (aus; indica) subspecies.

\section{Results}

\section{Chilling Tolerance Trait Phenotyping of RDP1 Accessions}

To better understand the natural variation that allows the JAPONICA subspecies of rice to be more chilling tolerant than the INDICA subspecies, multiple phenotypic analyses on a subset of Rice Diversity Panel 1 (RDP1; Eizenga et al. 2014) accessions were done at five different temperatures $\left(4^{\circ} \mathrm{C}, 8{ }^{\circ} \mathrm{C}, 10^{\circ} \mathrm{C}, 12^{\circ} \mathrm{C}\right.$, and $\left.16^{\circ} \mathrm{C}\right)$. The core subset of 354 out of 424 RDP1 accessions was chosen due to their consistently robust germination rates and abundant seed availability, and because their genotypes were included in the High-Density Rice Array (HDRA) containing 700,000 single nucleotide polymorphisms (SNPs; Chen et al. 2014). A principal component analysis using the SNP information showed that there was indeed considerable genetic variation that led to a clustering of the different subpopulations, which is a critical requirement for genome wide association studies (GWAS) and quantitative trait loci (QTL) mapping (Fig. 1). The rationale for choosing different chilling temperatures was to determine whether different stress tolerance pathways are active at different temperatures, or whether common pathways can be identified that are active at overlapping temperatures. The low-temperature seedling survivability (LTSS) phenotypic assay was chosen, because it was previously shown that at $10^{\circ} \mathrm{C}$, it significantly discriminated between accessions with different cold stress tolerance potentials (Schläppi et al. 2017). The electrolyte leakage (EL) phenotypic assay was chosen as a measure of plasma membrane integrity after cold temperature stress exposure, and it was previously shown that it can identify accessions with different cold stress tolerance potentials even when no differences in LTSS scores are observable (Shi et al. 2020). Phenotypic assays for EL and LTSS are shown in Fig. 2. Lastly, the median lethal chilling temperature (LT50), that is, the lethal temperature at which $50 \%$ of two-week-old seedlings die and $50 \%$ live after 1 week of cold exposure was calculated from LTSS scores to potentially identify additional chilling stress tolerance pathways at the threshold of survivability.

Collectively, as shown in Fig. 3 for three temperatures $\left(8{ }^{\circ} \mathrm{C}, 10^{\circ} \mathrm{C}\right.$, and $\left.12{ }^{\circ} \mathrm{C}\right)$, the JAPONICA and INDICA subspecies had significantly different LTSS, EL, and LT50 scores. Accessions within a particular subpopulation (aromatic, aus, indica, temperate japonica, tropical japonica, admixed JAPONICA, admixed INDICA, and admixed INDICA/JAPONICA) had similar chilling tolerance phenotypes, and they followed similar trajectories at every temperature tested. Interestingly, while most members of the JAPONICA subspecies were more chilling tolerant than members of the INDICA subspecies, aromatic accessions which are typically classified as part of the JAPONICA subspecies had chilling tolerance phenotypes that were more similar to admixed INDICA/

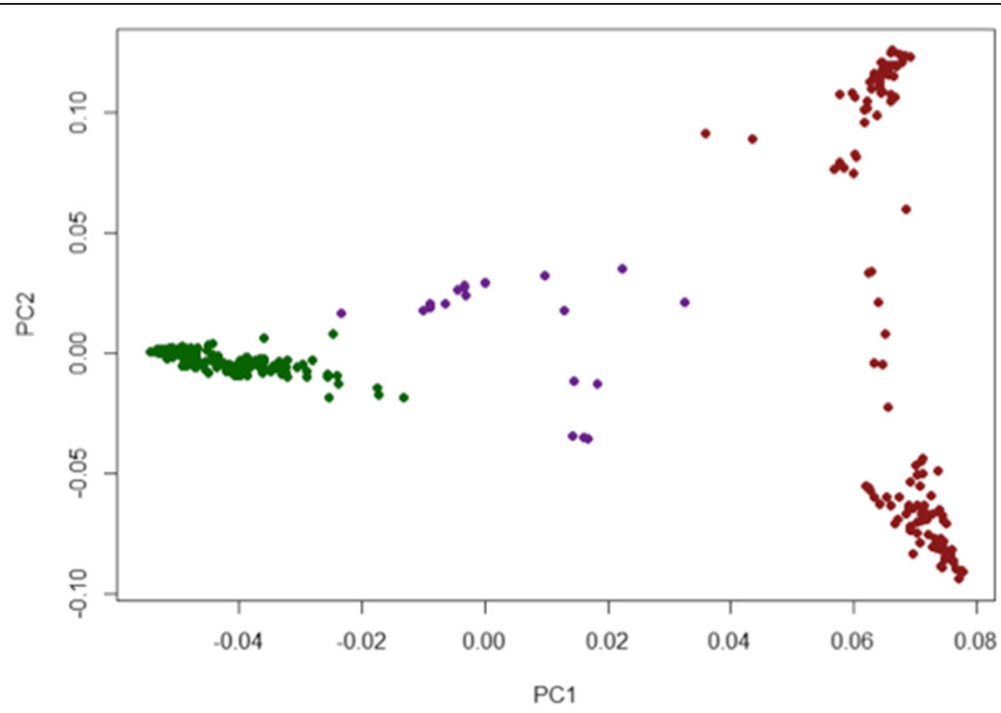

Fig. 1 Principle Component Analysis (PCA) to illustrate genotype-based justification for rice accession subgroup clustering based on chilling tolerance phenotypes. PCA was calculated using the $700 \mathrm{~K}$ HDRA SNP profiles of 354 Rice Diversity Panel 1 accessions. Green, Cold Tolerant cluster (temperate japonica; tropical japonica, japonica admixes; 205 accessions). Purple, Intermediate Cold Tolerant cluster (aromatic; indicajaponica admixes; 20 accessions). Red, Cold Sensitive cluster (aus, various indica; aus-indica admixes; 129 accessions) 


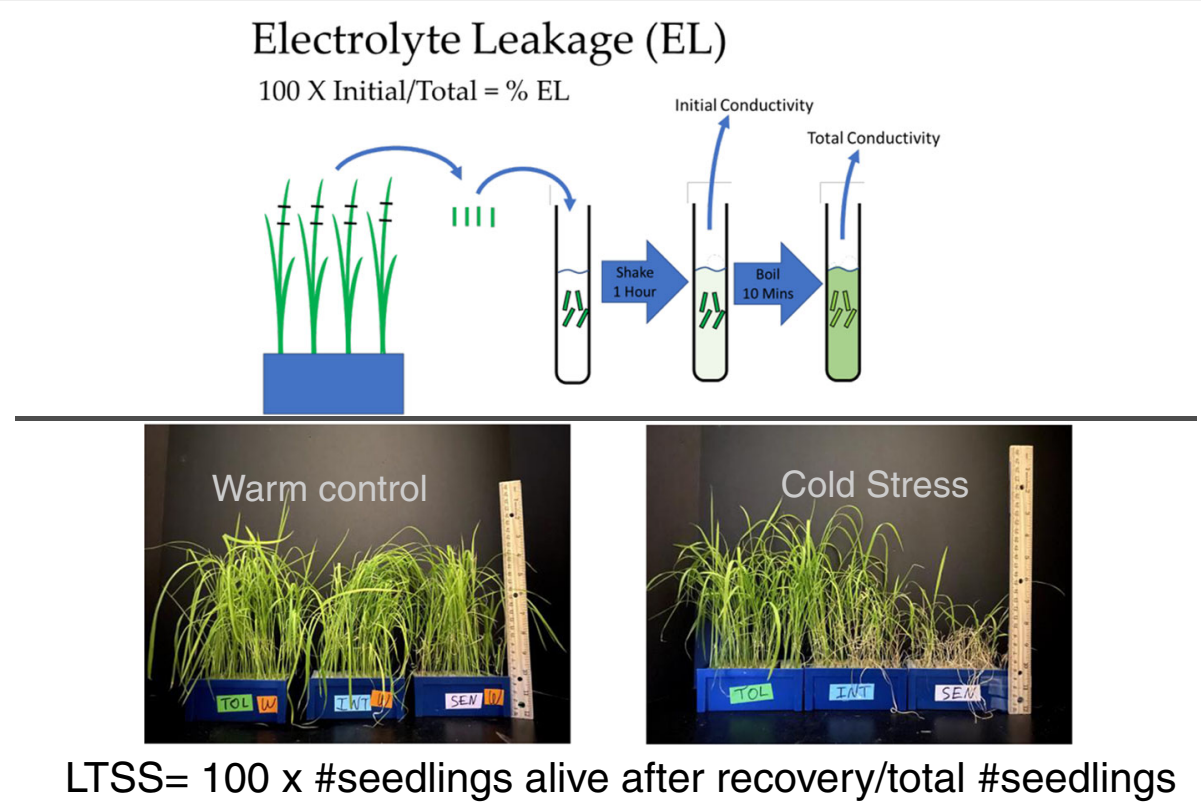

Fig. 2 Electrolyte Leakage (EL) and Low-temperature Seedling Survivability (LTSS) phenotype analyses. Upper panel: EL assays were done using middle sections of leaves from two-week-old seedlings exposed continuously to $10^{\circ} \mathrm{C}$ for 1 week. Lower panel: Left side, phenotype of threeweek-old rice seedlings grown at warm (W) temperatures. Right side, phenotype of two-week-old seedlings exposed continuously to $10^{\circ} \mathrm{C}$ for 1 week and after a one-week recovery period at warm temperatures. Most Col Tolerant (TOL) accessions are green and healthy looking. Most Cold Sensitive (SEN) accession are bleached and wrinkled. Intermediate (INT) Col Tolerant accessions have an intermediate phenotype

JAPONICA than to other JAPONICA relatives, most likely because genotypically they also clustered with admixed varieties (Fig. 1). In agreement with our results, it was previously observed that aromatic accessions clustered with admixed instead of temperate or tropical japonica accessions (Wang et al. 2018). For this reason, aromatic and admixed INDICA/JAPONICA accessions were grouped into a third "Intermediate Cold Tolerant" (Intermediate) cluster, distinct from the "Cold Tolerant" (Tolerant; temperate japonica, tropical japonica, and admixed JAPON$I C A$ ) and "Cold Sensitive" (Sensitive; aus, indica, and admixed INDICA) clusters. Admixed JAPONICA and admixed INDICA accessions predictably had phenotypes in accordance with their JAPONICA and INDICA mixed genotypes, respectively. However, it is interesting to note that admixed JAPONICA accessions were generally a bit less chilling tolerant than either temperate or tropical japonica accession while admixed INDICA accessions were generally a bit more chilling sensitive than either aus or indica accessions (Fig. 3). The three "chilling tolerance clusters" were used for multiple chilling tolerance trait analyses, which was the basis for subsequent GWAS mapping.

\section{Summary of Percent Electrolyte Leakage (EL) Results}

The percent EL was determined for each cultivar as a measure of plasma membrane integrity after cold temperature stress exposure, with the general expectation that cold sensitive accessions will release more electrolytes (high EL scores) from leaf tissues than cold tolerant accession (low EL scores), due to a higher incidence of membrane lesions after cold exposure. The mean percent EL for Tolerant, Intermediate, and Sensitive clusters generally followed this expectation (Fig. 4). For the Tolerant cluster, the mean percent EL at the five temperatures ranged from 8.7 to $20.9 \%$; for the Intermediate cluster, 9.4 to $34.3 \%$; and for the Sensitive cluster, 12.8 to $35.4 \%$. At each temperature, mean EL values were significantly lower for the Tolerant cluster than for the Sensitive clusters. Mean EL values were also significantly lower for the Tolerant cluster than the Intermediate cluster at $4{ }^{\circ} \mathrm{C}$ and $8^{\circ} \mathrm{C}$, however, there were no significant differences between 10 and $16^{\circ} \mathrm{C}$. This indicates that below $10^{\circ} \mathrm{C}$, the Intermediate cluster shifts from a more tolerant to a more sensitive EL phenotype. For the Sensitive cluster, similar EL values at $4{ }^{\circ} \mathrm{C}$ and $8{ }^{\circ} \mathrm{C}$ suggests that $8{ }^{\circ} \mathrm{C}$ is a threshold temperature for Sensitive accessions below which lower temperatures do not significantly increase membrane lesions any more. Taken together, these EL results suggest that Tolerant, Intermediate, and Sensitive clusters have different low-temperature thresholds at which maximum membrane lesions occur, and that each cluster has a different low-temperature buffering range for maintenance of membrane integrity.

\section{Summary of Low-Temperature Seedling Survivability (LTSS) Results}

The percent LTSS was determined as a measure of survival of two-week-old seedlings after 1 week of continuous 


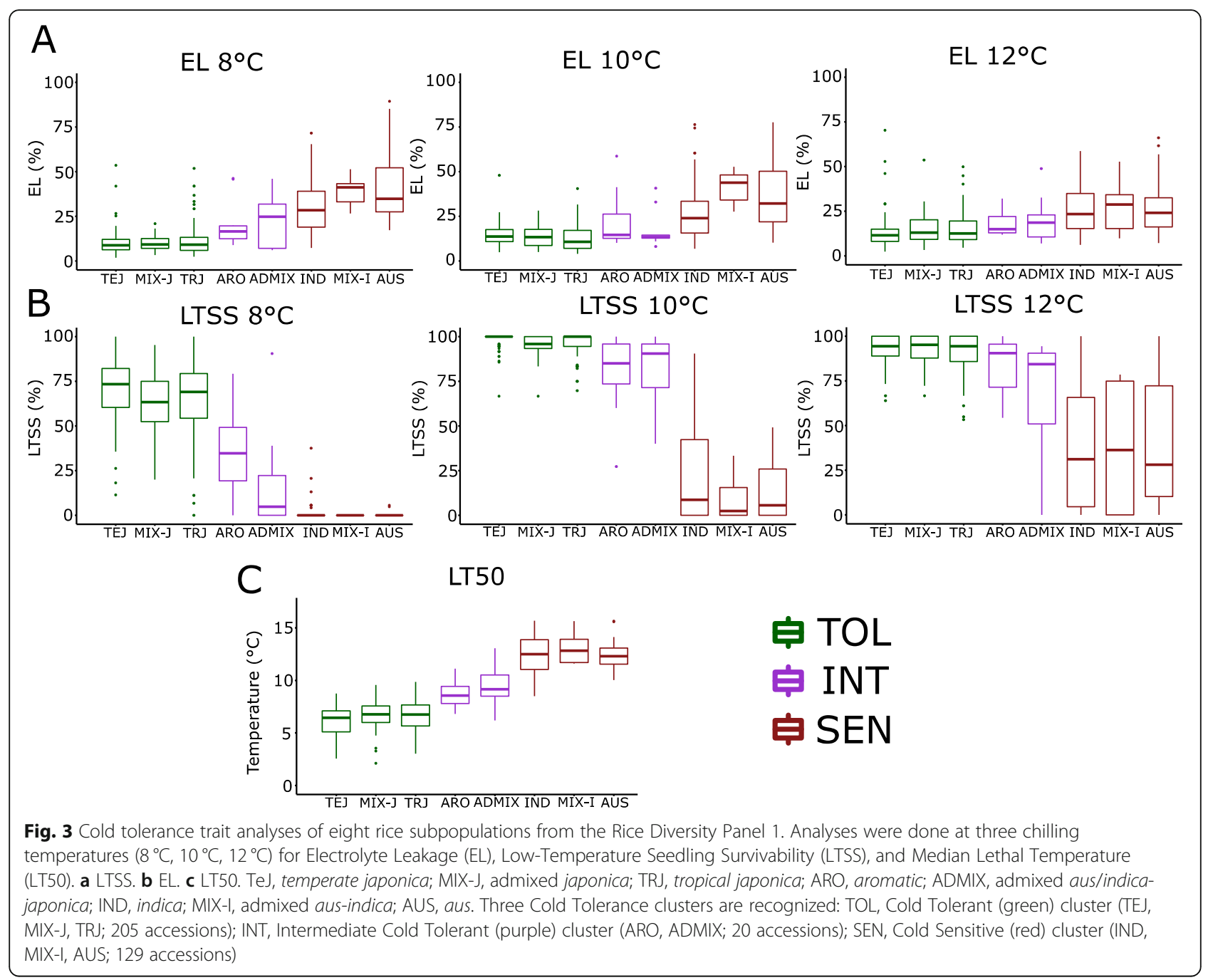

low-temperature stress exposure. The phenotype of plants after a 7-day recovery period from cold stress is shown in Fig. 2: recovered seedlings were alive, green, and healthy looking while dead seedlings were bleached and wilted. Our expectation was that the Tolerant cluster will have significantly more accessions with high LTSS scores than the Intermediate and Sensitive clusters. The mean percent LTSS for Tolerant, Intermediate, and Sensitive clusters generally followed this expectation (Fig. 5). For the Tolerant cluster, the mean percent LTSS at the five temperatures ranged from 20.1 to $98.2 \%$; for the Intermediate cluster, 3.0 to $96.2 \%$; and for the Sensitive cluster, 0.3 to 94.9\%. At each temperature, mean LTSS scores were significantly lower for the Sensitive cluster than for the other two clusters, and except for $16^{\circ} \mathrm{C}$, mean LTSS values were significantly higher for the Tolerant cluster than the Intermediate cluster. Between 4 and $10^{\circ} \mathrm{C}$, mean LTSS values for the Sensitive cluster were very similar, indicating that $10^{\circ} \mathrm{C}$ might be a threshold temperature for Sensitive accessions below which lower temperatures do not significantly decrease seedling survivability, which is similar to what was observed for the EL phenotype. Between 8 and $10{ }^{\circ} \mathrm{C}$, LTSS values for the Intermediate cluster shifted from a more tolerant to a more sensitive phenotype, which is also similar to what was observed for the EL phenotype, however, an apparent threshold for survivability was reached between 4 and $8^{\circ} \mathrm{C}$. The somewhat different temperature threshold buffering ranges for mean EL and LTSS scores suggests that there may not be a linear correlation between membrane integrity and seedling survivability.

\section{Correlation between LTSS and EL at Different Low Temperatures}

Percent EL and LTSS phenotypes of the Intermediate cluster were similar to those of the Tolerant and Sensitive clusters at high and low temperatures, respectively, but at different threshold temperatures (Figs. 4 and 5). This suggests that the EL phenotype, measured immediately after 1 week of low-temperature stress exposure, 

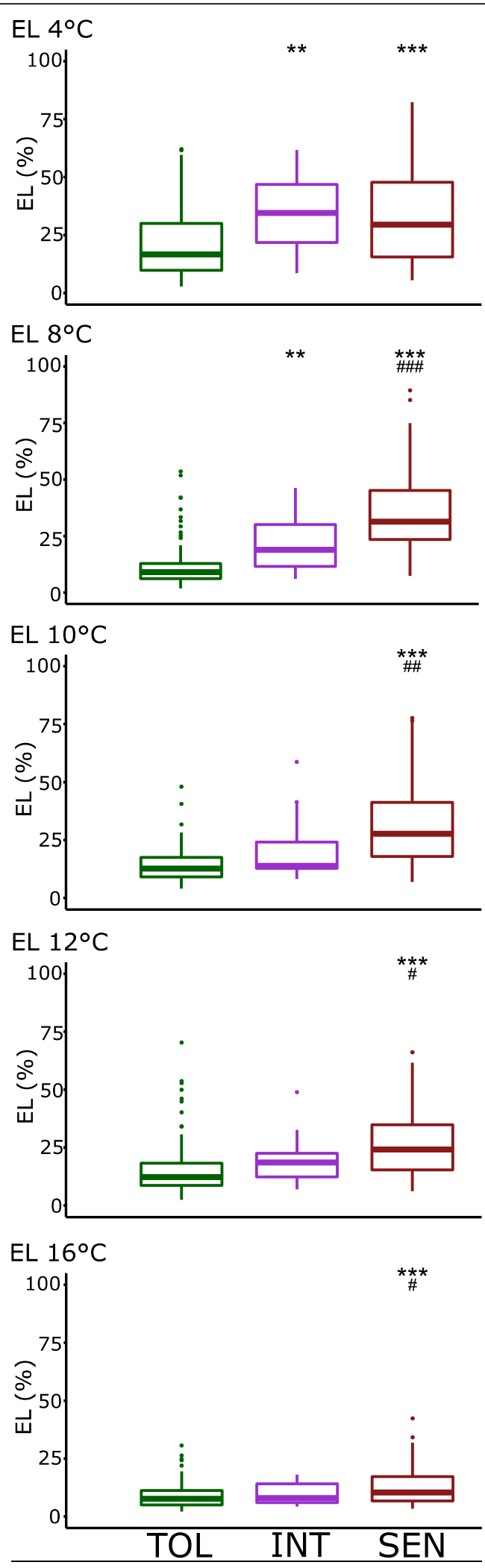

Fig. 4 Box plots of mean Electrolyte Leakage (EL) values at different chilling temperatures for rice accessions in each cold tolerance cluster. Significant differences compared to the Cold Tolerant (TOL) cluster at all temperatures is labeled by ${ }^{*}$. Significant differences compared to the Intermediate (INT) Col Tolerant cluster is labeled by \#. SEN, Cold Sensitive Cluster. ${ }^{* * *} p<0.001 ;{ }^{* *} p<0.01 ;{ }^{*} p<0.05 .{ }^{\# \# \#} p<$ $0.001 ;{ }^{\# \#} p<0.01 ;{ }^{\#} p<0.05$

might not accurately predict survival rates 1 week after recovery at growth promoting temperatures. Therefore, correlations between EL and LTSS were done to determine how effectively EL scores can be used to predict LTSS. Interestingly, linear correlation coefficients varied between the five temperatures (Fig. 6). The best correlation was observed at $8^{\circ} \mathrm{C}\left(R^{2}=0.4620\right)$, followed by $10^{\circ} \mathrm{C} \quad\left(R^{2}=0.3696\right)$ and $12^{\circ} \mathrm{C} \quad\left(R^{2}=\right.$ $0.2869)$, while there was no correlation at the extreme ends of $4{ }^{\circ} \mathrm{C} \quad\left(R^{2}=0.0746\right)$ and $16^{\circ} \mathrm{C} \quad\left(R^{2}=0.0098\right)$. This indicates that at $8{ }^{\circ} \mathrm{C} \pm 1{ }^{\circ} \mathrm{C}$, the degree of chilling temperature induced membrane damage correlates well with subsequent probability for survival. That is, less membrane damage predicts higher survival rates, however, even at $8{ }^{\circ} \mathrm{C}$, there were several outliers with little membrane damage and low survival rates, and several outliers with a high degree of membrane damage and high survival rates (Fig. 6). This suggests that for some accessions, membrane damage sustained during cold exposure is not the main cause for low survival rates, indicating that physiological or metabolic events during the recovery phase affect survival rates. In contrast, even though significant membrane damage occurs during cold exposure, some accessions can deal with this damage during the recovery phase, leading to increased survival rates.

\section{Summary of Median Lethal Chilling Temperature (LT50) Results}

Our data show that different "cold tolerance clusters" (Figs. 4 and 5) as well as different subgroups (Fig. 3) had different "tipping points" where EL or LTSS scores changed dramatically. To include these tipping points as an additional parameter for GWAS analyses, we calculated LT50 values from LTSS data for each accession at which $50 \%$ of seedlings died and $50 \%$ survived. Using $R$, LTSS curves were generated by plotting median LTSS rates at $4{ }^{\circ} \mathrm{C}, 8{ }^{\circ} \mathrm{C}, 10^{\circ} \mathrm{C}, 12^{\circ} \mathrm{C}$, and $16^{\circ} \mathrm{C}$ to identify LT50 for each accession (Fig. 7a). The curves were clustered for Tolerant, Intermediate, and Sensitive accessions, and median LT50 were calculated for each cluster (Fig. 7b). The survival curves and median LT50 values for each tolerance cluster generally followed the patterns observed for LTSS, further supporting our classification of aromatic accessions as an Intermediate cluster. 

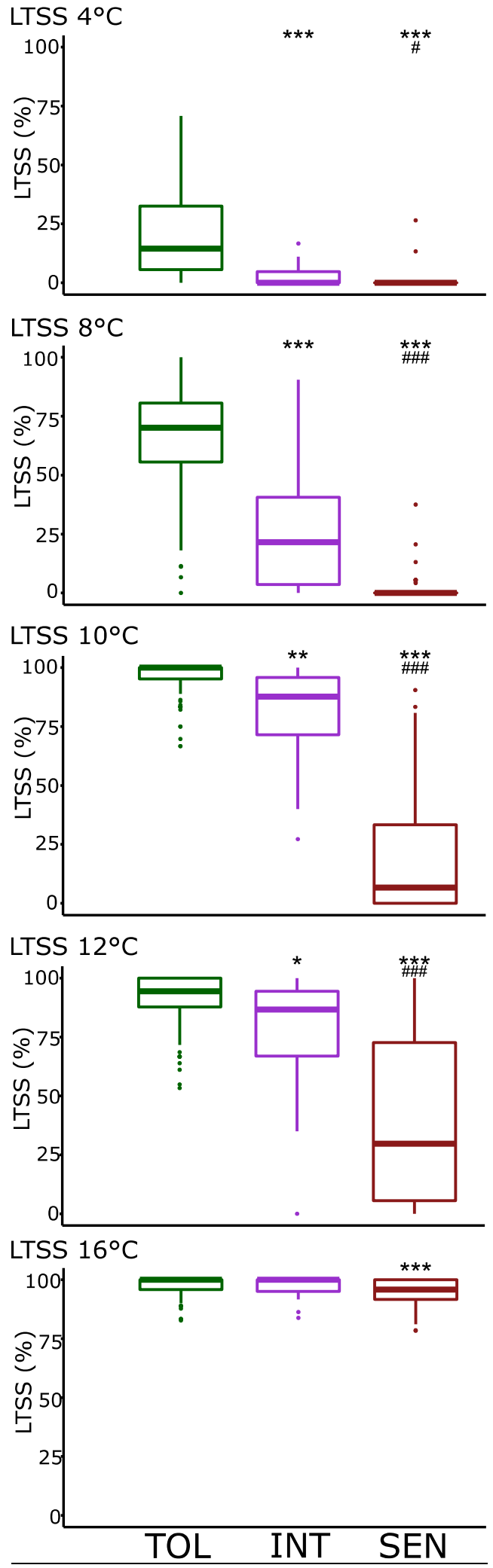

Fig. 5 Box plots of mean Low-Temperature Seedling Survivability (LTSS) values at different chilling temperatures for rice accessions in each cold tolerance cluster. Significant differences compared to the Cold Tolerant (TOL) cluster at all temperatures is labeled by *. Significant differences compared to the Intermediate (INT) Col Tolerant cluster is labeled by \#. SEN, Cold Sensitive Cluster. ${ }^{* * *} p<$ $0.001 ;{ }^{* *} p<0.01 ;{ }^{*} p<0.05 .{ }^{\# \# \#} p<0.001 ;{ }^{\# \#} p<0.01 ;{ }^{\#} p<0.05$

Genome-Wide Association Study (GWAS)-Based Mapping of EL, LTSS, and LT50 QTL

From the core subset of 354 RDP1 accessions, EL, LTSS, and LT50 data were used to perform GWAS to identify significantly trait associated SNPs. We applied a publicly available GWAS pipeline (McCouch et al. 2016) that accounted for population structure of the rice accessions using a kinship matrix created by the efficient mixedmodel association eXpedited (EMMAX) approach (Kang et al. 2010), which determined ancestry or cryptic relatedness between individual genotyping markers. GWAS-mapping pipeline produced quantile-quantile (Q-Q) plots and Manhattan plots for each cold tolerance trait are shown in Fig. S1 and Fig. 8, respectively. Although several Q-Q plots appeared to indicate inflation in some of the phenotypes, our plots had similar trends as those of the original mapping results reported in the study that established the RDP1 mapping pipeline (McCouch et al. 2016). Therefore, based on Q-Q plots a $p$-value cutoff for significant SNPs was determined to be at $-\log 10(p)>4$, and QTL were identified as chromosomal sites containing 3 significant SNPs within a one million base pair (Mbp) region.

Across the three traits and five temperatures, 245 total QTL were identified. The LTSS trait yielded 150 QTL, the EL trait 92 QTL, and the LT50 trait 3 QTL (Table S1-S7). Temperature also affected the number of QTL identified. The moderate chilling temperatures of $10{ }^{\circ} \mathrm{C}$ and $12{ }^{\circ} \mathrm{C}$ yielded 83 and 62 QTL covering $11.6 \%$ and $8.9 \%$ of the rice genome, respectively, while $4{ }^{\circ} \mathrm{C}, 8{ }^{\circ} \mathrm{C}$, and $16^{\circ} \mathrm{C}$ yielded 38,29 , and 30 QTL respectively (Table S1-S5). Many of the 245 QTL were found in overlapping chromosomal regions, some of which were longer than 1 Mbp (Fig. 9; Fig. S2). Taken together, we identified 178 unique QTL, consisting of 40 multiple-trait QTL ( $q M T)$ and 138 single-trait QTL ( $q E$ for $q E L$, and $q L$ for $q L T S S$ ) (Table S7), covering approximately $25 \%$ of the rice genome.

Using different cold tolerance clusters, additional GWAS-based QTL mappings were done. To identify QTL that control chilling tolerance in accessions of the Tolerant and Sensitive clusters, the Intermediate cluster was removed and GWAS mappings were done for the three high EL-LTSS-correlation temperatures of $8{ }^{\circ} \mathrm{C}$, $10{ }^{\circ} \mathrm{C}$, and $12^{\circ} \mathrm{C}$ (Table S8). GWAS mapping was also done within the Tolerant and Sensitive clusters to 

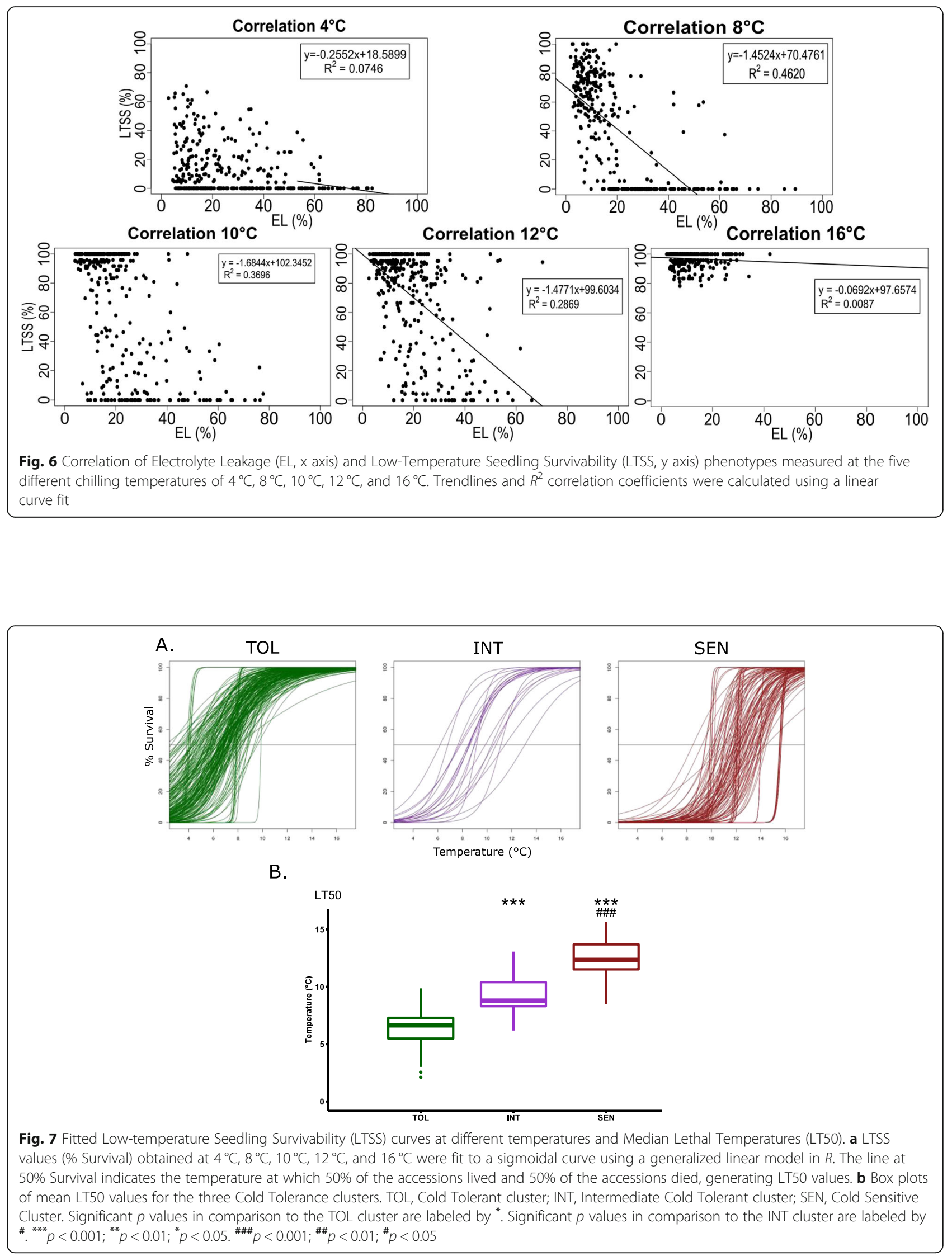


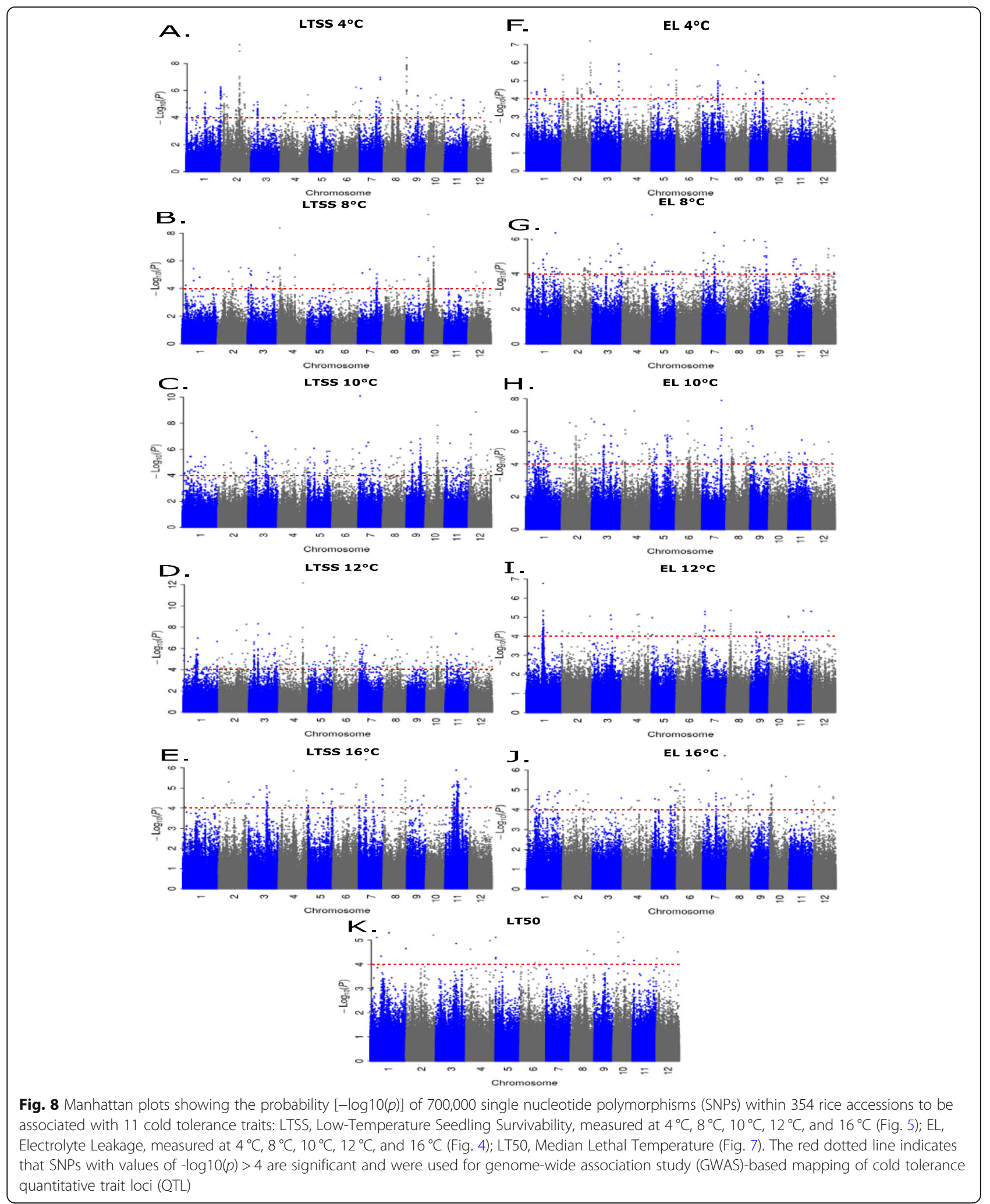

identify QTL correlating with phenotypic variability within each subspecies for the three high EL-LTSS-correlation temperatures of $8{ }^{\circ} \mathrm{C}, 10^{\circ} \mathrm{C}$, and $12^{\circ} \mathrm{C}$ (Table S9). The
Tolerant versus Sensitive (TvS) GWAS mapping resulted in 219 individual QTL: 68 EL QTL, 150 LTSS QTL, and 1 LT50 QTL. The within-subspecies GWAS mapping 


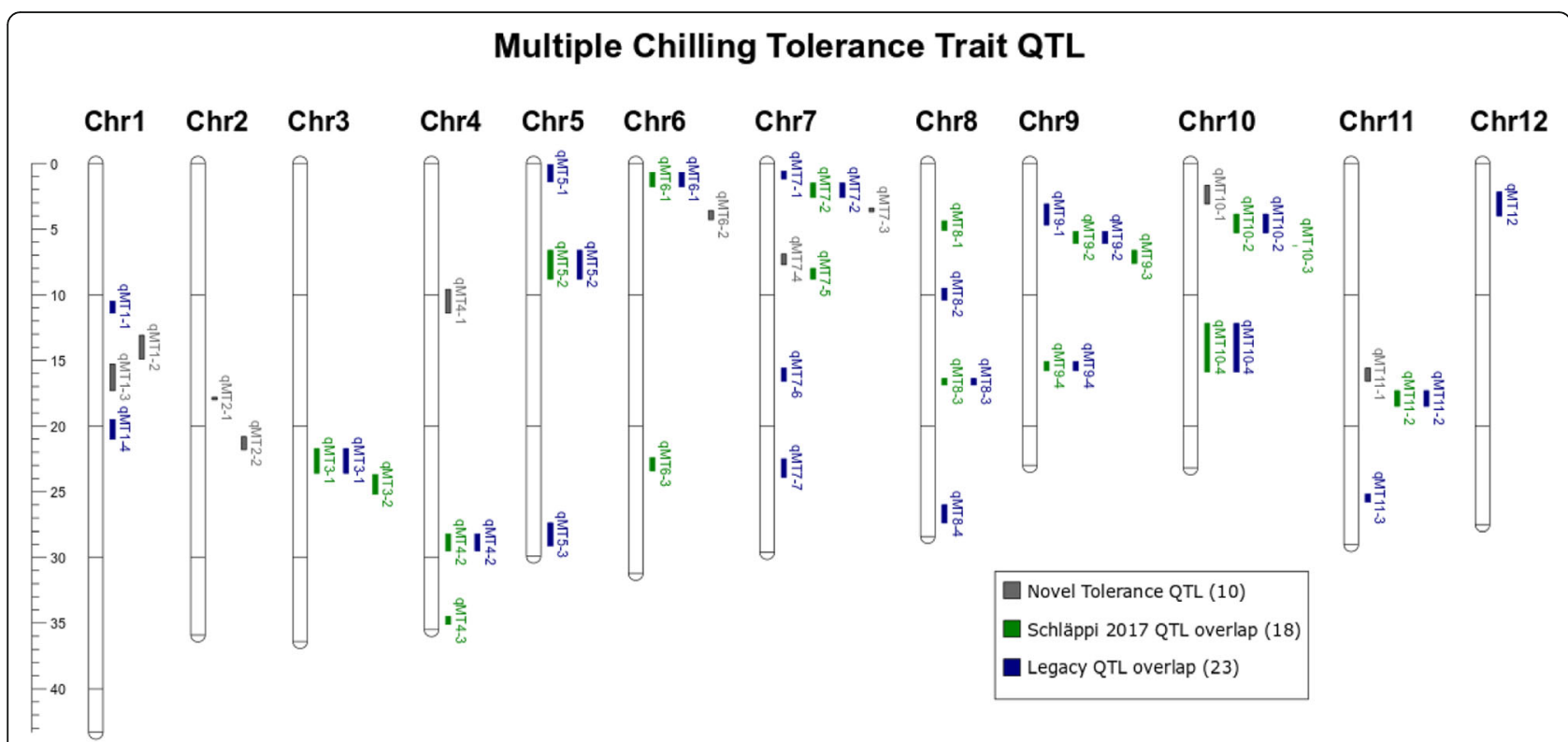

Fig. 9 Genomic locations of 40 Multiple-Trait QTL ( $(M M T)$. Bars show the genomic regions of GMT QTL covering at least two of the following individual trait QTL: Electrolyte Leakage (EL) measured at $4{ }^{\circ} \mathrm{C}, 8^{\circ} \mathrm{C}, 10^{\circ} \mathrm{C}, 12^{\circ} \mathrm{C}$, or $16^{\circ} \mathrm{C}$ (Fig. 4); Low-Temperature Seedling Survivability (LTSS) measured at $4^{\circ} \mathrm{C}, 8^{\circ} \mathrm{C}, 10^{\circ} \mathrm{C}, 12^{\circ} \mathrm{C}$, or $16^{\circ} \mathrm{C}$ (Fig. 5); and Median Lethal Temperature (LT50, Fig. 7). Grey bars show novel seedling stage cold tolerance QTL identified in this study, while green bars show qMT overlapping with various seedling stage cold tolerance QTL identified by Schläppi et al. 2017, and blue bars show 9MT overlapping with legacy seedling stage cold tolerance QTL identified by Andaya and Mackill 2003; Liu et al. 2013; Ma et al. 2015; Lv et al. 2016; and Wang et al. 2016

resulted in 36 individual QTL: Mapping within the Sensitive cluster yielded 33 QTL consisting of 3 EL QTL, 29 LTSS QTL, and 1 LT50 QTL; and mapping within the Tolerant cluster yielded 3 QTL consisting of 2 EL QTL and 1 LTSS QTL. This result is consistent with the larger genetic variation within Sensitive (Fig. 1, red cluster) than Tolerant (Fig. 1, green cluster) accessions.

Within the 178 unique QTL regions obtained using all 354 accessions, 14,710 genes were identified, which is more than $25 \%$ of the total number of annotated genes reported by the Rice Genome Annotation Project (Kawahara et al. 2013). Since a major focus of our GWAS analysis was to identify general cold tolerance genes with natural variation between chilling tolerant and chilling sensitive rice accessions and to reduce the list of potential candidates, we interrogated the 40 qMT QTL regions for chilling tolerance candidate genes (Table 1). There were 30 QTL that overlapped with previously published cold tolerance QTL (Andaya and Mackill 2003; Liu et al. 2013; Ma et al. 2015; Wang et al. 2016; Lv et al. 2016; Schläppi et al. 2017). Genes found in the $q M T$ regions are hypothesized to be involved in either multiple traits (EL, LTSS, LT50) or in helping with improving those traits at more than one temperature. This reduced the total number of genes to be analyzed from 14,710 to 6065 genes associated with the $40 q M T$ regions, which were further analyzed using gene function and Gene Ontology (GO) term annotations.
To identify cold tolerance candidate genes within the $q M T$ QTL, annotated genes that contained significant SNPs $\left(p<10^{-4}\right)$ within their genomic regions were selected, yielding a list of 220 genes. Significant SNPs with expected percentages of major alleles and direction of allele effect on chilling tolerance trait were used to further filter the gene list. That is, significant SNPs with a major allele frequency at least as much as the percentage of accessions of the Tolerant cluster (205 out of 354 accessions $=58 \%$ ) and with an allele effect positively correlating with the measured chilling tolerance trait (to decrease EL and to increase LTSS) were selected, yielding a list of 81 candidate genes. Those genes were then used for GO term enrichment analysis and compared to the larger gene list to narrow down possible cold tolerance response pathways. Furthermore, since GWASbased QTL mapping was also done using accessions from between and within the major cold Tolerant and Sensitive clusters, $35 q M T$ QTL regions with at least one single QTL using these clusters were identified and used for targeted GO enrichment analysis (Table 1). In addition to using a kinship matrix, this analysis was done to reduce the potential influence of population structure in defining significant regions. Thus, the rationale for using regions that were highlighted in mappings within clusters was to account for a potential population structure bias arising from only the differences in variability between the Sensitive and Tolerant clusters. Those 35 
Table 1 Table of Multiple Trait QTL

\begin{tabular}{|c|c|c|c|c|c|c|c|c|}
\hline$\overline{\mathrm{QTL}}$ & Chr & Start & End & Mapping Phenotypes & $\begin{array}{l}\text { Cluster } \\
\text { Overlap }\end{array}$ & $\begin{array}{l}\text { Peak } \\
\text { LOD }\end{array}$ & Peak SNP gene & Published QTL \\
\hline qMT1-1 & 1 & $\begin{array}{l}10,527 \\
625\end{array}$ & $\begin{array}{l}11,389 \\
874\end{array}$ & $\mathrm{E} 10+\mathrm{L} 10$ & TVS & 5.444609 & & $\operatorname{Locus}^{a}$ \\
\hline qMT1-2 & 1 & $\begin{array}{l}13,105 \\
151\end{array}$ & $\begin{array}{l}14,895 \\
303\end{array}$ & $\mathrm{E} 10+\mathrm{E} 16+\mathrm{L} 12$ & TvS & 4.769976 & & \\
\hline qMT1-3 & 1 & $\begin{array}{l}15,272 \\
806\end{array}$ & $\begin{array}{l}17,301 \\
559\end{array}$ & $\mathrm{E} 10+\mathrm{L} 12$ & TvS & 5.906907 & & \\
\hline qMT1-4 & 1 & $\begin{array}{l}19,518 \\
259\end{array}$ & $\begin{array}{l}20,986 \\
910\end{array}$ & $\mathrm{E} 8+\mathrm{E} 12$ & TvS & 6.768674 & & Locus $4^{a}$, aCTS $1-3^{c}$ \\
\hline qMT2-1 & 2 & $\begin{array}{l}17,820 \\
486\end{array}$ & $\begin{array}{l}18,028 \\
915\end{array}$ & $\mathrm{~L} 4+\mathrm{L} 8$ & & 4.680601 & $\begin{array}{l}\text { LOC_ } \\
\text { Os02g30270 }\end{array}$ & \\
\hline qMT2-2 & 2 & $\begin{array}{l}20,805 \\
063\end{array}$ & $\begin{array}{l}21,772 \\
969\end{array}$ & $\mathrm{E} 10+\mathrm{L} 4$ & TvS & 7.292885 & & \\
\hline qMT3-1 & 3 & $\begin{array}{l}21,728 \\
962\end{array}$ & $\begin{array}{l}23,622 \\
225\end{array}$ & $\begin{array}{l}\mathrm{E} 10+\mathrm{E} 12+\mathrm{L} 8+\mathrm{L} 10+\mathrm{L} 12+ \\
\mathrm{L} 16\end{array}$ & $S+T v S$ & 6.272784 & & qCTS3-11 c \\
\hline qMT3-2 & 3 & $\begin{array}{l}23,729 \\
655\end{array}$ & $\begin{array}{l}25,203 \\
390\end{array}$ & $\mathrm{E} 10+\mathrm{L} 10+\mathrm{L} 12$ & $S+T v S$ & 7.380266 & & aLTSS3-3/aLTS3-2 ${ }^{b}$ \\
\hline qMT4-1 & 4 & $9,599,400$ & $\begin{array}{l}11,394 \\
804\end{array}$ & $\mathrm{~L} 10+\mathrm{L} 12$ & TVS & 6.484325 & & \\
\hline qMT4-2 & 4 & $\begin{array}{l}28,197 \\
218\end{array}$ & $\begin{array}{l}29,473 \\
755\end{array}$ & $\mathrm{E} 10+\mathrm{L} 12$ & TvS & 12.14515 & & LoCUS47 ${ }^{a}$, aLTSS4-2 $2^{b}$, aCTS4-3 ${ }^{e}$ \\
\hline qMT4-3 & 4 & $\begin{array}{l}34,467 \\
694\end{array}$ & $\begin{array}{l}35,089 \\
971\end{array}$ & $\mathrm{E} 4+\mathrm{L} 10$ & $S+T v S$ & 6.47215 & $\begin{array}{l}\mathrm{LOC}_{-} \\
\text {Os04g58780 }\end{array}$ & aLTSS4-3/aLTS4 ${ }^{b}$ \\
\hline qMT5-1 & 5 & 69,986 & $1,409,302$ & $\mathrm{E} 8+\mathrm{L} 10+\mathrm{L} 16+\mathrm{LT} 50$ & TvS & 7.38114 & & Locus $51^{a}$ \\
\hline qMT5-2 & 5 & $6,600,746$ & $8,783,967$ & $\mathrm{E} 10+\mathrm{L} 10+\mathrm{L} 12$ & TVS & 6.324189 & & $\operatorname{aLTS5} 5^{b}, \operatorname{aCTS5}-3^{c}$ \\
\hline qMT5-3 & 5 & $\begin{array}{l}27,413 \\
208\end{array}$ & $\begin{array}{l}29,075 \\
588\end{array}$ & $\mathrm{E} 16+\mathrm{L} 16$ & & 4.955528 & $\begin{array}{l}\text { LOC_ } \\
\text { Os05g48960 }\end{array}$ & Locus $56^{a}$ \\
\hline qMT6-1 & 6 & 690,949 & $1,804,604$ & $\mathrm{E} 16+\mathrm{L} 12$ & $S+T v S$ & 7.250662 & $\begin{array}{l}\text { LOC_ } \\
\text { Os06g02710 }\end{array}$ & Locus $57^{a}, a^{P G C 6-1}{ }^{b}$ \\
\hline qMT6-2 & 6 & $3,567,142$ & $4,288,870$ & $\mathrm{E} 16+\mathrm{L} 12$ & TVS & 5.286129 & $\begin{array}{l}\text { LOC_ } \\
\text { Os06g07420 }\end{array}$ & \\
\hline qMT6-3 & 6 & $\begin{array}{l}22,367 \\
941\end{array}$ & $\begin{array}{l}23,364 \\
932\end{array}$ & $\mathrm{E} 10+\mathrm{L} 4+\mathrm{L} 12$ & & 5.466931 & & qLTG6 ${ }^{b}$ \\
\hline qMT7-1 & 7 & 592,074 & $1,198,046$ & $\mathrm{~L} 12+\mathrm{L} 16$ & TVS & 7.1194 & & aCTS7-1 ${ }^{c}$ \\
\hline qMT7-2 & 7 & $1,452,372$ & $2,559,101$ & $\mathrm{E} 8+\mathrm{L} 10$ & $S+T v S$ & 10.08297 & & LocUS69 ${ }^{a}$, aLTSS7-1 ${ }^{b}$ \\
\hline qMT7-3 & 7 & $3,402,347$ & $3,683,917$ & $\mathrm{E} 12+\mathrm{L} 12$ & TVS & 6.412739 & $\begin{array}{l}\text { LOC_ } \\
\text { Os07g07240 }\end{array}$ & \\
\hline qMT7-4 & 7 & $6,870,947$ & $7,663,360$ & $\mathrm{E} 16+\mathrm{L} 12$ & TVS & 5.970675 & $\begin{array}{l}\text { LOC_- } \\
\text { Os07g12330 }\end{array}$ & \\
\hline qMT7-5 & 7 & $7,997,910$ & $8,776,532$ & $\mathrm{~L} 12+\mathrm{L} 16$ & TvS & 6.408 & & aLTSS7-2 ${ }^{b}$ \\
\hline qMT7-6 & 7 & $\begin{array}{l}15,627 \\
934\end{array}$ & $\begin{array}{l}16,632 \\
053\end{array}$ & $\mathrm{E} 16+\mathrm{L} 10$ & $S+T v S$ & 5.094292 & & LoCUS75 ${ }^{a}$, qCTS $^{d}$ \\
\hline qMT7-7 & 7 & $\begin{array}{l}22,505 \\
330\end{array}$ & $\begin{array}{l}23,930 \\
131\end{array}$ & $\mathrm{E} 10+\mathrm{L} 8$ & TvS & 7.906038 & & qCTS $7-4^{c}$ \\
\hline qMT8-1 & 8 & $4,409,763$ & $5,060,164$ & $\mathrm{E} 12+\mathrm{L} 12$ & TVS & 7.134518 & & $a L S S 8-1^{b}$ \\
\hline qMT8-2 & 8 & $9,468,694$ & $\begin{array}{l}10,399 \\
998\end{array}$ & $\mathrm{~L} 4+\mathrm{L} 12$ & TvS & 7.793283 & & qCTS $8-2^{c}$ \\
\hline qMT8-3 & 8 & $\begin{array}{l}16,369 \\
660\end{array}$ & $\begin{array}{l}16,896 \\
911\end{array}$ & $\mathrm{~L} 4+\mathrm{L} 12$ & TvS & 6.393616 & $\begin{array}{l}\text { LOC_ } \\
\text { Os08g27240 }\end{array}$ & $\operatorname{LoCUS}_{8}{ }^{a}$, aLTSS8-2/qLTS8 $^{b}$ \\
\hline qMT8-4 & 8 & $\begin{array}{l}26,014 \\
290\end{array}$ & $\begin{array}{l}27,371 \\
604\end{array}$ & $\mathrm{E} 8+\mathrm{E} 10+\mathrm{L} 16$ & TvS & 5.37007 & $\begin{array}{l}\text { LOC_ } \\
\text { Os08g41830 }\end{array}$ & $\operatorname{Locus}_{8} 7^{a}, \mathrm{COLD}^{f}$ \\
\hline qMT9-1 & 9 & $3,149,137$ & $4,733,475$ & $\mathrm{E} 8+\mathrm{E} 10$ & TVS & 6.185335 & & LoCUS91 ${ }^{a}$, aCTS9-3/aCTS9-4/aCTS9-5 \\
\hline
\end{tabular}


Table 1 Table of Multiple Trait QTL (Continued)

\begin{tabular}{|c|c|c|c|c|c|c|c|c|}
\hline$\overline{\mathrm{QTL}}$ & Chr & Start & End & Mapping Phenotypes & $\begin{array}{l}\text { Cluster } \\
\text { Overlap }\end{array}$ & $\begin{array}{l}\text { Peak } \\
\text { LOD }\end{array}$ & Peak SNP gene & Published QTL \\
\hline qMT9-2 & 9 & $5,186,978$ & $6,076,169$ & $\mathrm{E} 8+\mathrm{E} 10$ & TVS & 8.264567 & & LoCUS93 $^{a}$, aPGCG9-1 $^{b}$ \\
\hline 9MT9-3 & 9 & $6,569,358$ & $7,630,726$ & $\mathrm{E} 4+\mathrm{L} 10$ & TvS & 5.145748 & & $a^{P} G C G 9-1{ }^{b}$ \\
\hline qMT9-4 & 9 & $\begin{array}{l}15,055 \\
972\end{array}$ & $\begin{array}{l}15,835 \\
730\end{array}$ & $\mathrm{E} 4+\mathrm{L} 10$ & TvS & 5.000415 & $\begin{array}{l}\text { LOC_ } \\
\text { Os09g25220 }\end{array}$ & $\begin{array}{l}\text { LoCUS97 } a, \text { aLTG-9 }{ }^{d}, \text { aPGCG9-2/ } \\
\text { qLTSS9-2 } b\end{array}$ \\
\hline $\begin{array}{l}\text { qMT10- } \\
1\end{array}$ & 10 & $1,740,269$ & $3,057,764$ & $\mathrm{E} 16+\mathrm{L} 10$ & TVS & 6.125558 & & \\
\hline $2^{\text {qMT10- }}$ & 10 & $3,909,466$ & $5,322,884$ & $\mathrm{~L} 8+\mathrm{L} 10$ & $S+T v S$ & 10.35381 & & Locus101 $a$, aLTSS10-1 ${ }^{b}$ \\
\hline $\begin{array}{l}\text { 9MT10- } \\
3\end{array}$ & 10 & $6,299,787$ & $6,300,600$ & $\mathrm{~L} 12+\mathrm{LT} 50$ & TvS & 7.180984 & $\begin{array}{l}\text { LOC_ } \\
\text { Os10g11354 }\end{array}$ & aLTSS10-1 ${ }^{b}$ \\
\hline $\begin{array}{l}\text { qMT10- } \\
4\end{array}$ & 10 & $\begin{array}{l}12,242 \\
058\end{array}$ & $\begin{array}{l}15,899 \\
908\end{array}$ & $\mathrm{~L} 10+\mathrm{L} 12+\mathrm{LT} 50$ & $S+T v S$ & 7.843522 & & LoCus104 ${ }^{a}$, aLTSS10-2 ${ }^{b}$ \\
\hline${ }_{1}^{\text {qMT11- }}$ & 11 & $\begin{array}{l}15,593 \\
585\end{array}$ & $\begin{array}{l}16,583 \\
082\end{array}$ & $\mathrm{~L} 12+\mathrm{L} 16$ & & 5.455106 & $\begin{array}{l}\text { LOC_ } \\
\text { Os11g28184 }\end{array}$ & \\
\hline $2^{9 M T 11-}$ & 11 & $\begin{array}{l}17,316 \\
314\end{array}$ & $\begin{array}{l}18,487 \\
194\end{array}$ & $\mathrm{E} 10+\mathrm{L} 12$ & $S+T v S$ & 5.02026 & $\begin{array}{l}\text { LOC_ } \\
\text { Os11g31620 }\end{array}$ & Locus $111^{a}$, aLTSS11-3 ${ }^{b}$, aCTS11-5 ${ }^{c}$ \\
\hline $\begin{array}{l}\text { qMT11- } \\
3\end{array}$ & 11 & $\begin{array}{l}25,159 \\
572\end{array}$ & $\begin{array}{l}25,809 \\
234\end{array}$ & $\mathrm{~L} 10+\mathrm{L} 12$ & & 5.266234 & & Locus $116^{a}$, aCTS11-10 \\
\hline qMT12 & 12 & $2,192,895$ & $4,043,986$ & $\mathrm{E} 8+\mathrm{L} 10+\mathrm{L} 12$ & $S+T v S$ & 8.710121 & $\begin{array}{l}\text { LOC_ } \\
\text { Os12g06270 }\end{array}$ & $\operatorname{Locus} 121^{a}$ \\
\hline
\end{tabular}

qMT Multi-Trait QTL containing overlapping individual QTL mapped with cold tolerance traits

E Electrolyte Leakage

L Low-Temperature Seedling Survivability

LT50: median low temperature of seedling survival

$4,8,10,12,16: 4{ }^{\circ} \mathrm{C}, 8^{\circ} \mathrm{C}, 10^{\circ} \mathrm{C}, 12{ }^{\circ} \mathrm{C}, 16^{\circ} \mathrm{C}$

TvS: Tolerant cluster vs Sensitive cluster OTL overlapping with $q M T$ OTL

$T$ Tolerant cluster QTL overlapping with qMT QTL

$S$ Sensitive cluster QTL overlapping with $q M T$ QTL

LOD: $-\log 10(p)$ values of peak SNPs within $q M T$ QTL

Peak SNP gene: gene within a qMT QTL containing a peak SNP

a Lv et al. 2016

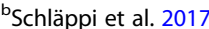

'Wang et al. 2016

d Liu et al. 2013

eAndaya and Mackill 2003

fMa et al. 2015

qMT QTL were further filtered for genes containing significant SNPs, which resulted in 207 genes that were separately used for GO term enrichment analysis.

\section{GO Term Analyses of all Genes within Multi-Trait ( $q M T$ ) \\ QTL}

The $q M T$ QTL gene list was purged of annotated transposable elements resulting in 4256 genes which were analyzed for GO term enrichment. GO term annotations were obtained for 2483 of the 4256 genes, and 257 GO terms were found to be enriched $(p<0.01)$, and those relevant to plant chilling tolerance were grouped into chilling stress relevant clusters. Within the GO term for Biological Process, three major clusters were identified for "carbohydrate derivative biosynthesis", "protein modification by small protein conjugation or removal", "carbohydrate transmembrane transport", and "hydrogen peroxide mediated signaling pathway" (Fig. S3). Within the GO term for Cell Component, there were only two clusters: "transferase complex" and "intrinsic component of plasma membrane" (Fig. S4). Within the GO term for Molecular Function, numerous clusters were identified including: "ADP binding", "carbohydrate transmembrane transporter activity", "oxidoreductase activity", "thioldependent ubiquitinyl hydrolase activity", and "ubiquitin-like protein transferase activity" (Fig. S5). These terms suggest possible associations with chilling tolerance mechanisms and can be used to select pools of genes for further analysis. These genes are expected to be involved in metabolic homeostasis, membrane composition, ubiquitination, oxidative stress, and signaling pathways.

\section{GO Term Analysis of Filtered Gene List from qMT QTL}

The filtered list of 81 genes containing significant SNPs was also purged of annotated transposable elements, reducing it to 71 genes for GO term enrichment analysis. Due to the low gene number, significance of enrichment was set to $p<0.05$. GO term annotations were obtained 
for 45 genes and 10 terms were enriched. In Biological Process, "cellular response to stress" and "transmembrane transport" were enriched (Fig. S6). In Cell Component, "Golgi membrane" and "extracellular region" were enriched (Fig. S7). In Molecular Function, "molecular transducer activity" and "exonuclease activity" were enriched (Fig. S8). These enrichment clusters suggest likely interaction of genes involved in vesicle transport and secretion and nucleic acid processing to confer cold stress tolerance responses. Enrichment for cellular response to stress and Golgi membrane suggest that these filtered genes may be involved in modifications and transport of membranes and membrane-bound proteins.

\section{GO Term Analyses of Significant Genes within Multiple- Trait and Cold Tolerant \& Cold Sensitive Cluster QTL} Genes within the 35 qMT QTL that overlapped with cold Tolerant and cold Sensitive cluster-specific QTL (Table 1) were separately investigated to identify enriched GO terms. Out of 67 input genes, the full filtering protocol resulted in only three enriched $(p<0.05)$ GO terms: "exonuclease activity", "cytoplasmic part", and "extracellular region". As term enrichment map visualizations were not possible with so few terms, an assessment was done on a larger list of genes from an intermediate filtering protocol. The intermediate filtering of only genes with significant SNPs resulted in 159 non-transposable element genes with significant SNPs from the $35 q M T$ and Tolerant \& Sensitive Cluster QTL. The terms that were enriched at this stage were related to "ubiquitination", "plant growth", and "nucleotide binding" (Fig. S9, S10 and S11).

\section{Discussion}

\section{Chilling Tolerance Phenotype of each Cluster is Differentially Affected by Stress Severity}

The percent low-temperature seedling survivability (LTSS) and percent electrolyte leakage (EL) means of different rice accessions at different chilling temperatures generally followed an expected trajectory based on their subpopulation structure, including the recent chilling tolerance reevaluation of aromatic and admixed accessions (Wang et al. 2018). That is, temperate and tropical japonica accessions and admixed japonicas, comprising the more chilling tolerant JAPONICA subspecies, generally had lower EL and higher LTSS scores than aus and indica accessions and aus/indica admixes, comprising the more chilling sensitive INDICA subspecies (Fig. 3). It is important to note that due to artificial human selection and geographic regions of cultivation, the JAPONICA subspecies is generally more cold tolerant than the INDICA subspecies, which is at least in part due to selection of specific transcription factor alleles, such as bZIP73, in the direct ancestor of japonica contributing to cold climate adaptation during early stages of rice domestication (Liu et al. 2018). Due to artificial human selection of other beneficial traits associated with warm climate adaptation, a trade-off between those traits and cold climate adaptation resulted in cold sensitivity of the INDICA subspecies, because there was no need to invest energy into genetic and metabolic pathways promoting cold tolerance.

Interestingly, we did not observe a good correlation between LTSS and EL at each chilling temperature, indicating that under certain low-temperature stress conditions, EL cannot be used to predict seedling survivability after stress recovery (Fig. 6). This lack of correlation might also suggest that at certain chilling temperatures, the degree of membrane damage sustained during stress exposure is not the only determinant for seedling survivability. That many data points deviated from the trendline even for the best EL-LTSS correlation at $8{ }^{\circ} \mathrm{C}$ suggests that there may be different cold tolerance response mechanisms controlling membrane integrity and survival in rice. For submergence stress, there are two major response mechanisms that rice uses to survive under hypoxia stress, including growth restriction and rapid growth and cell elongation (Septiningsih et al. 2009; Nagai et al. 2010; Miro and Ismail 2013). Analogous to this, there may be alternative or even competing stress tolerance response mechanisms that different cold tolerant accessions use under the same or varying lowtemperature stress conditions.

\section{Genome-Wide Association Study (GWAS)-Based Mapping} of EL, LTSS, and LT50 QTL

The 11 cold tolerance traits [(EL + LTSS $) \times 5$ temperatures + LT50] we assessed yielded 245 quantitative trait loci (QTL) using 354 rice diversity panel 1 (RDP1) accessions comprising of all five major subpopulations of rice (aromatic, aus, indica, temperate japonica, tropical japonica) and some admixes between them. Because standard regression techniques used to perform association studies assume that all variables within a data set are mutually independent, genetic relatedness between individuals in a population can generate false positive associations (Sul et al. 2018). To correct for this, the publicly available GWAS pipeline we employed uses a mixed model based on EMMAX. We are, therefore, confident that the single nucleotide polymorphism (SNP) variants we identified as associated with the 11 cold tolerance traits are within a $95 \%$ confidence interval, because mixed models were previously shown in rice and humans to adequately correct for population structure in the sample (McCouch et al. 2016; Sul et al. 2018). Performing additional GWAS analyses using different subsets of accessions yielded another 255 QTL, many of which overlapped with the 245 QTL using all accessions. Together, the 500 QTL identified 178 unique 
chromosomal regions covering $25 \%$ of the rice genome, indicating that chilling tolerance in rice is a multigenic trait. Interestingly, 36 out of 39 (92\%) seedling stage cold tolerance QTL we previously identified using the Rice Mini-Core collection (Schläppi et al. 2017) were validated by at least one QTL identified using RDP1 accessions, and 18 out of $39(46 \%)$ were validated by multiple-trait QTL ( $q M T$; Fig. 9; Table 1). Moreover, at least 23 out of 40 (57.5\%) qMT QTL overlapped with legacy rice seedling stage cold tolerance QTL identified during the last 20 years (Fig. 9; Table 1). Taken together, the validation of many cold tolerance QTL that were previously identified using different collections of rice accessions suggests that the QTL shown here are robust and adequate for the identification of associated stress tolerance response genes.

\section{Chilling Stress-Relevant Gene Ontology (GO) Term Clusters}

The GO term enrichment and clustering data suggested that the major GO term clusters for the genes found within the multiple-trait QTL have a high likelihood to be involved in different mechanisms of cold stress tolerance responses. In the clusters determined by the filtered list of 71 genes, some specific pathways emerged. "Golgi apparatus" as a significant cluster suggests an involvement of membrane transport from the endoplasmic reticulum (ER) to other locations in the cell. Together with GO term clusters derived from larger multiple-trait QTL genes, this suggests that the Golgi apparatus where ubiquitination determines the destination of different Golgi-associated proteins has an important function in regulating cold stress tolerance responses. It was previously shown that ubiquitination of proteins in the Golgi can be used as a signal for subcellular localization to many destinations, including, the nucleus, the vacuole, and the plasma membrane (Risinger and Kaiser 2008; Scheuring et al. 2012; Stone 2014; Liu and Li 2019). Ubiquitination and the Golgi apparatus are also involved in plant hormone signaling pathways (Stone 2014). These known interactions suggest that some of the candidate genes found in the filtered 71 gene list are involved in a Golgi-mediated cold stress response mechanism.

In addition to its intracellular localization function, the Golgi apparatus is involved in the secretory pathway for many cellular components. The GO enrichment in both the full multiple-trait QTL gene list and the filtered 71-gene list uncovered terms relevant to transmembrane transport. Specifically, the full-gene list enrichment resulted in term clusters of genes involved in both carbohydrate biosynthesis and carbohydrate transmembrane transport. This could be due to a difference in the ability of Tolerant and Sensitive clusters to manage osmotic homeostasis under chilling stress, an important component of chilling tolerance (Afzal et al. 2016).
As the filtered gene list pointed to the involvement of the Golgi and ubiquitination, the pathways may converge in the process of subcellular localization or extracellular secretion of critical components for cell wall modification and longrange signaling (Park et al. 2017; Takahashi et al. 2019). These findings suggest that one of the chilling stress tolerance response mechanisms that is different between the $J A$ PONICA and INDICA subspecies may be how the Golgi apparatus responds or functions during chilling stress.

A third class of candidate genes are associated with terms of signal transduction. Many GO term clusters or cluster members involved "nucleotide binding", "signal", and "protein modification by small molecules". These terms suggest that differences in signal transduction pathways might explain cold tolerance differences between Tolerant and Sensitive accessions. These genes could be involved in transducing signals for phytohormones, mechanosensory channels, plasma membrane ion channels, or relaying secondary signaling events throughout the cell to plastids in response to chilling stress.

Finally, the term "regulation of plant growth and development" appeared multiple times in enrichments for different gene populations. The most obvious function for genes in this cluster is related to how plant growth and development is regulated during chilling stress. Analogous to the aforementioned submergence tolerance, one cold stress tolerance response mechanism might lead to complete growth cessation while another one might lead to cellular and metabolic homeostasis allowing a certain degree of growth and development even under cold temperatures. Interactions between growth-regulating genes and phytohormones are well known (Nagai et al. 2010; Colebrook et al. 2014; Sah et al. 2016), and hormones such as abscisic acid (ABA) have previously been linked to abiotic stress, including cold tolerance (Lado and Manzi 2017; Gibson 2004; Sah et al. 2016; Liu et al. 2018).

\section{Conclusions}

This study provides novel insight into the potential mechanisms for cold tolerance in rice using GWAS-based mapping of two chilling tolerance traits under different lowtemperature stress conditions. We show that at different chilling temperatures, different potential mechanisms might control cold stress tolerance responses to reduce plasma membrane damage and enhance seedling survivability. Interestingly, the highest $\left(16^{\circ} \mathrm{C}\right)$ and lowest $\left(4^{\circ} \mathrm{C}\right)$ temperatures tested had the least correlation between membrane damage and seedling survivability, suggesting differences in mechanisms involved in survival at these temperatures compared to $8-12{ }^{\circ} \mathrm{C}$. The 500 QTL controlling membrane integrity and seedling survivability under chilling stress identified in this study cover approximately $25 \%$ of the rice genome, while the 40 multiple-trait QTL among them cover approximately $10 \%$ of the rice genome. This is in agreement with the notion 
that cold tolerance is a complex and polygenic trait. Genes within those genomic regions were enriched for terms involved in "carbohydrate biosynthesis and transmembrane transport", "plasma membrane", "ubiquitination", and "ADP binding". Among them, 71 genes with significant SNPs and allele effects were enriched for "Golgi apparatus", "transmembrane transport", "signal transduction", and "stress response". These enrichments suggest a likely involvement of the secretory and subcellular localization functions of the Golgi apparatus. With the introduction of QTL derived from cold tolerance cluster-specific mapping, the common themes of "plant growth", "ubiquitination", "signaling", and "transmembrane transport" were highlighted. These terms, along with "Golgi apparatus", suggest a difference between Tolerant and Sensitive accessions in the function of signaling pathways, possibly involving hormones such as ABA (Fig. S12). Investigation of candidate genes involved in or regulating these pathways is a critical next step in understanding the mechanistic differences in chilling stress tolerance responses between accessions from the JAPONICA and INDICA subspecies of rice.

\section{Methods}

\section{Plant Materials}

Seeds from 354 rice accessions were obtained from the USDA/ARS Genetics Stocks-Oryza (GSOR) collection. All of the accessions were all part of the Rice Diversity Panel 1 (RDP1) that were genotyped using the high-density rice array (HDRA) containing 700,000 (700 K) single nucleotide polymorphisms (SNPs; Eizenga et al. 2014; McCouch et al. 2016). These lines were used as a representation of the entire Oryza sativa species to map genotypic variances that explain the subspecies differences in chilling tolerance levels between JAPONICA and INDICA.

\section{Germination and Standard Growth Conditions}

Seeds were germinated in tap water at $37^{\circ} \mathrm{C}$ in the dark for 3 days in petri dishes with $0.1 \%$ bleach solution to prevent bacterial growth. Germinating seeds were moved to hydroponics boxes after 2 days of germination for the final day of germination to prevent root overgrowth and maintain the same direction of shoot growth. The hydroponics boxes were filled with deionized (DI) water with no bleach. After germination, the hydroponics boxes were moved to a chamber with standard growth conditions of $12 \mathrm{~h}$ light $/ 12 \mathrm{~h}$ dark cycles and $28{ }^{\circ} \mathrm{C} / 25^{\circ} \mathrm{C}$ day/night temperatures. The germinated seeds were allowed to grow until 2weeks of age. At 10 days post germination, the water in the boxes were replaced with 1/4-strength Murashige-Skoog basal salt medium (Murashige and Skoog 1962). Each accession was represented by up to 8 plants per box in a triplicate randomized block design for up to 24 plants (3 boxes) per experiment.

\section{Chilling Stress Treatment}

Two-week-old, seedlings were transferred for 7 days to a low-temperature growth chamber with a $12 \mathrm{~h} / 12 \mathrm{~h}$ light/ dark cycle and a constant chilling temperature. Watering was adjusted to a minimum due to significantly lower water depletion in boxes and an effort to minimize chamber temperature fluctuations. Five chilling temperatures were tested independently: $4^{\circ} \mathrm{C}, 8{ }^{\circ} \mathrm{C}, 10^{\circ} \mathrm{C}, 12^{\circ} \mathrm{C}$, and $16^{\circ} \mathrm{C}$. At the end of the 7 -day period, leaf samples were taken for electrolyte leakage (EL) measurements and plants were returned to standard growth conditions $(12 \mathrm{~h}$ light $/ 12 \mathrm{~h}$ dark, $28^{\circ} \mathrm{C} / 25^{\circ} \mathrm{C}$ day/night cycles) after replacement of hydroponic solution with fresh $1 / 4 \times$ MS media.

\section{Recovery}

Plants were allowed to recover for 7 days in normal growth conditions, with watering at least every other day. Low-temperature seedling survivability (LTSS) was recorded, as described below, on day 7 .

\section{EL and LTSS Chilling Tolerance Trait Phenotyping}

EL was measured after 7 days of chilling stress treatment. For each cultivar, tissue segments were cut from the center of four leaves, washed in DI water, and pooled into glass screw-cap tubes containing $5 \mathrm{~mL}$ of DI water having at a conductivity of no more than $1 \mu \mathrm{S} / \mathrm{cm}$. The tubes were shaken for $1 \mathrm{~h}$ and initial conductivity was measured twice using a LAQUA twin B-771 hand-held conductivity meter (HORIBA Scientific, Japan). The tubes were then boiled for $10 \mathrm{~min}$, shaken for $10 \mathrm{~min}$, and allowed to cool. The total conductivity was measured from cooled tubes. Mean percent EL was then calculated as \%EL $=[($ initial EL divided by total EL) times 100]. Replicate EL values were averaged, and that average was used for quantitative trait loci (QTL) mapping.

LTSS was measured after 7 days of recovery. Alive and dead seedlings were determined by visual inspection as previously described (Schläppi et al. 2017): alive were green and healthy looking; dead were bleached and wilted. The mean percent survivability was calculated as \%LTSS $=[$ (number of green and healthy-looking seedlings after 1 week of recovery growth divided by the number of initial healthy-looking seedlings before treatment) times 100]. Replicate LTSS values were averaged, and that average was used for QTL mapping.

Median lethal temperature (LT50) was calculated using dead and alive counts from LTSS recordings of all five temperatures analyzed. LTSS curves as a function of temperature were calculated using a generalized linear model using a binomial distribution in $R$ ( $\mathrm{R}$ Core Team 2018). The $R$ script used was derived from https://lukemiller.org/index.php/201 0/02/calculating-lt50-median-lethal-temperature-aka-ld50quickly-in-r/ (Miller 2010). 


\section{Genome-Wide Association Study (GWAS)-Based QTL Mapping}

Cold stress tolerance phenotyping was done on a subset of 354 out of 424 RDP1 accessions that were used for genotyping with the $700 \mathrm{~K}$ SNPs of the HDRA (Chen et al. 2014; McCouch et al. 2016). A GWAS mapping python pipeline for this subset of RDP1, created by McCouch et al. 2016, was used for GWAS-based QTL mapping and generating Manhattan and quantile-quantile (Q-Q) plots. The pipeline uses a variance component approach implemented in the publicly available software, efficient (linear) mixed-model association eXpedited (EMMAX; Kang et al. 2010), to map HDRA-derived $700 \mathrm{~K}$ SNPs to phenotype and to account for population structure by calculating and including a kinship matrix as a covariate, which helps to distinguish true associations from false positives due to relatedness between accessions. The kinship matrix was calculated using identity by state (IBS) to group identical allele states while excluding shared ancestry. The default allele filtering settings were used to run the pipeline: minor allele frequency (MAF) $>10 \%$, minor allele count $(\mathrm{MAC})>1$, percent missing allele $<30 \%$.

\section{Quantitative Trait Loci (QTL)}

QTL were identified using a protocol similar to one used by Shakiba et al. 2017. SNPs were determined significant if they had $-\log 10(p)>4$, because most of the Q-Q plots diverged from expected near 4 (Fig. S1). QTL were assigned when 3 or more significant SNPs were found within a $1 \mathrm{Mb}$ region. Eleven phenotypes were used for GWAS-based QTL mapping: 5 sets of EL data from 5 temperatures tested, 5 sets of LTSS data from 5 temperatures tested, and the calculated LT50 for each of the 354 accessions.

\section{Candidate Gene Identification and Gene Ontology (GO) Term Analysis}

Genes within QTL were identified using the Allele Finder web tool provided by the Rice Diversity Project (McCouch et al. 2016). All genes found within a QTL were deemed potential candidate genes. GO term enrichments of all genes identified within QTL regions were determined using the GO Enrichment Analysis web tool from http:// plantregmap.cbi.pku.edu.cn. GO term clustering and enrichment visualization was done by using the REVIGO webtool from http://revigo.irb.hr (Supek et al. 2011).

\section{Candidate Gene Filtering}

Genes were filtered based on expected mechanistic involvement in chilling tolerance due to GO term annotation as well as tissue and stress responsive expression data provided by the Rice Genome Annotation Project from Michigan State University (Kawahara et al. 2013). Using gene descriptions, transposable elements were filtered out to focus the search on protein encoding genes likely to be involved in chilling tolerance responses. GO enrichment analysis was conducted on these genes to identify likely functional enrichment as a guide to understanding possible cold tolerance mechanisms. The transposon-free list of genes was also filtered based on the presence of significant SNPs identified by GWAS mapping to find genes with SNP variations that resulted in significant effects on chilling tolerance traits. These genes were further filtered to identify SNPs with major allele distribution relatively close to the proportion of accessions belonging to the Tolerant cluster (205 out of 354 accessions $=58 \%$ ) to identify genes involved in mechanisms underlying cluster-specific chilling tolerance phenotypes.

\section{Statistical Analysis}

Significant differences for all cold tolerance phenotypes were calculated using an $R$ computation of Welch's ANOVA and a Games-Howell non-parametric post hoc test due to significant differences in sample size as well as violation of the assumption of normality when comparing data between accession clusters.

\section{Supplementary information}

Supplementary information accompanies this paper at https://doi.org/10. 1186/s12284-020-00414-3.

Additional file 1 Figure S1. Quantile-Quantile (Q-Q) plots for GWAS mapping.

Additional file 2 Figure S2. Approximate genomic locations of 40 Multiple-Trait ( $q M T)$ QTL.

Additional file 3 Figure S3. Multiple-Trait QTL gene Biological Processes enrichment map.

Additional file 4 Figure S4. Multiple-Trait QTL gene Cell Component enrichment map.

Additional file 5 Figure S5. Multiple-Trait QTL gene Molecular Function enrichment map.

Additional file 6 Figure S6. Filtered Significant SNP Biological Processes enrichment map.

Additional file 7 Figure S7. Filtered Significant SNP Cell Component enrichment map.

Additional file $\mathbf{8}$ Figure S8. Filtered Significant SNP Molecular Function enrichment map.

Additional file 9 Figure S9. Multiple-Trait+Cluster QTL Biological Process enrichment map.

Additional file 10 Figure S10. Multiple-Trait+Cluster QTL Cell Component enrichment map.

Additional file 11 Figure S11. Multiple-Trait+Cluster QTL Molecular Function enrichment map.

Additional file 12 Figure S12: Model for functional associations of candidate term clusters.

Additional file 13 Tables S1-S10. QTL positions resulting from various GWAS mappings.

\section{Abbreviations}

ABA: Abscisic acid; EL: Electrolyte leakage; EMMAX: Efficient mixed-model association eXpedited; GWAS: Genome-wide association study/ies; HDRA: High density rice array; IBS: Identity by state; LT50: Median lethal chilling temperature; LTSS: Low temperature seedling survivability; Mbp: One million base pair; Q-Q: Quantile-quantile; QTL: Quantitative trait locus/i; qE: EL QTL; 
qL: LTSS QTL; qMT: Multiple-trait QTL; RDP1: Rice diversity panel 1; SNP: Single nucleotide polymorphism(s); TvS: Tolerant versus Sensitive

\section{Acknowledgements}

We are grateful to Marquette University undergraduate students Jacob Hess, Yicel Lechuga, and Marchell Waight who helped perform phenotyping trials as part of independent studies or summer research experiences. Lorie Bernhardt, USDA-ARS DBNRRC GSOR, is acknowledged for her technical expertise in preparing and shipping seed of the RDP1 accessions.

\section{Authors' Contributions}

MS and NS conceived the project and designed the experiments. NS, AB, and MJ performed the experiments. NS and MS performed all data analyses and wrote the manuscript. All authors read and approved the final manuscript.

\section{Funding}

NS and MS were supported in part by Bridge Funding from Marquette University and by NIFA-AFRI grant No. 2016-67013-24587 from the United States Department of Agriculture. MS, AB and MJ were supported in part by Strategic Innovation Fund SF\#105-17 from Marquette University.

\section{Availability of Data and Materials}

All data generated or analyzed during this study are included in this article and its supplementary information files.

\section{Ethics Approval and Consent to Participate}

Not applicable.

\section{Consent for Publication}

Not applicable.

\section{Competing Interests}

The authors declare that they have no competing interests.

Received: 30 March 2020 Accepted: 29 July 2020

Published online: 14 August 2020

\section{References}

Afzal Z, Howton T, Sun Y, Mukhtar M (2016) The roles of aquaporins in plant stress responses. J Dev Biol 4. p. 9. https://doi.org/10.3390/jdb4010009

Andaya VC, Mackill DJ (2003) Mapping of QTLs associated with cold tolerance during the vegetative stage in rice. J Exp Bot 54:2579-2585. https://doi.org/ 10.1093/jxb/erg243

Chen $\mathrm{H}$, Xie W, He H et al (2014) A high-density SNP genotyping Array for Rice biology and molecular breeding. Mol Plant 7:541-553. https://doi.org/10. $1093 / \mathrm{mp} / \mathrm{sst} 135$

Colebrook EH, Thomas SG, Phillips AL, Hedden P (2014) The role of gibberellin signalling in plant responses to abiotic stress. J Exp Biol 217. p. 67

da Cruz RP, Sperotto RA, Cargnelutti D et al (2013) Avoiding damage and achieving cold tolerance in rice plants. Food Energy Secur 2:96-119

Eizenga GC, Ali ML, Bryant RJ et al (2014) Registration of the Rice diversity panel 1 for genomewide association studies. J Plant Regist 8:109. https://doi.org/10. 3198/jpr2013.03.0013crmp

Food and Agriculture Organization of the United Nations (FAO) (2017) The future of food and agriculture: Trends and challenges. Food and Agriculture Organization of the United Nations, Rome

Gibson SI (2004) Sugar and phytohormone response pathways: navigating a signalling network. J Exp Bot 55:253-264. https://doi.org/10.1093/jxb/erh048

Kang HM, Sul JH, Service SK et al (2010) Variance component model to account for sample structure in genome-wide association studies. Nat Genet 42:348354. https://doi.org/10.1038/ng.548

Kawahara Y, de la Bastide M, Hamilton JP et al (2013) Improvement of the Oryza sativa Nipponbare reference genome using next generation sequence and optical map data. Rice 6:4. https://doi.org/10.1186/1939-8433-6-4

Lado J, Manzi M (2017) Metabolic and hormonal responses of plants to cold stress. In: Medicinal plants and environmental challenges. Springer International Publishing, Cham, pp 137-158

Liu C, Ou C, Mao B, Tang J, Wang W, Wang H, Cao S, Schläppi MR, Zhao B, Xiao G, Wang X, Chu C (2018) Early selection of bZIP73 facilitated adaptaion of japonica rice to cold climates. Nature Comm 9:3302. https://doi.org/10.1038/ s41467-018-05753-w
Liu F, Xu W, Song Q et al (2013) Microarray-assisted fine-mapping of quantitative trait loci for cold tolerance in rice. Mol Plant 6:757-767. https://doi.org/10.1093/mp/sss161

Liu L, Li J (2019) Communications between the endoplasmic reticulum and other organelles during abiotic stress response in plants. Front Plant Sci 10. https:// doi.org/10.3389/fpls.2019.00749

LV Y, Guo Z, Li X et al (2016) New insights into the genetic basis of natural chilling and cold shock tolerance in rice by genome-wide association analysis. Plant Cell Environ 39:556-570. https://doi.org/10.1111/pce.12635

Ma Y, Dai X, Xu Y et al (2015) COLD1 confers chilling tolerance in rice. Cell 160: 1209-1221. https://doi.org/10.1016/j.cell.2015.01.046

McCouch SR, Wright MH, Tung C-W et al (2016) Open access resources for genome-wide association mapping in rice. Nat Commun 7:10532. https://doi. org/10.1038/ncomms10532

McLean J, Hardy B, Hettel G (2013) Rice almanac, 4th edn

Miller L (2010) Calculating LT50 (median lethal temperature, aka LD50) quickly in R. https://lukemiller.org/index.php/2010/02/calculating-lt50-median-lethaltemperature-aka-ld50-quickly-in-r/

Miro B, Ismail AM (2013) Tolerance of anaerobic conditions caused by flooding during germination and early growth in rice (Oryza sativa L.). Front Plant Sci 4:269. https://doi.org/10.3389/fpls.2013.00269

Murashige T, Skoog F (1962) A revised medium for rapid growth and bio assays with tobacco tissue cultures. Physiol Plant 15:473-497. https://doi.org/10. 1111/j.1399-3054.1962.tb08052.x

Nagai K, Hattori Y, Ashikari M (2010) Stunt or elongate? Two opposite strategies by which rice adapts to floods. J Plant Res 123:303-309

Park J, Lee Y, Martinoia E, Geisler M (2017) Plant hormone transporters: what we know and what we would like to know. BMC Biol 15. p. 93

R Core Team (2018) R: A Language and Environment for Statistical Computing

Risinger AL, Kaiser CA (2008) Different ubiquitin signals act at the Golgi and plasma membrane to direct GAP1 trafficking. Mol Biol Cell 19:2962-2972. https://doi.org/10.1091/mbc.e07-06-0627

Sah SK, Reddy KR, Li J (2016) Abscisic acid and abiotic stress tolerance in crop plants. Front Plant Sci 7. p. 571. https://doi.org/10.3389/fpls.2016.00571

Scheuring D, Künzl F, Viotti C et al (2012) Ubiquitin initiates sorting of Golgi and plasma membrane proteins into the vacuolar degradation pathway. BMC Plant Biol 12. https://doi.org/10.1186/1471-2229-12-164

Schläppi MR, Jackson AK, Eizenga GC et al (2017) Assessment of five chilling tolerance traits and GWAS mapping in Rice using the USDA mini-Core collection. Front Plant Sci 8:957. https://doi.org/10.3389/fpls.2017.00957

Septiningsih EM, Pamplona AM, Sanchez DL et al (2009) Development of submergence-tolerant rice cultivars: the Sub1 locus and beyond. Ann Bot 103:151-160. https://doi.org/10.1093/aob/men206

Shakiba E, Edwards JD, Jodari F et al (2017) Genetic architecture of cold tolerance in rice (Oryza sativa) determined through high resolution genome-wide analysis. PLoS One 12:e0172133. https://doi.org/10.1371/journal.pone.0172133

Shi Y, Phan H, Liu Y et al (2020) Glycosyltransferase OsUGT90A1 helps protect the plasma membrane during chilling stress in rice. J Exp Bot 71:2723-2739. https://doi.org/10.1093/jxb/eraa025

Singh BK, Sutradhar M, Singh AK, Mandal N (2017) Cold stress in Rice at early growth stage - an overview. J Pure App Biosci 5:407-419. https://doi.org/10. 18782/2320-7051.2750

Stone SL (2014) The role of ubiquitin and the 265 proteasome in plant abiotic stress signaling. Front Plant Sci 5:1-10. https://doi.org/10.3389/fpls.2014.00135

Sul JH, Martin LS, Eskin E (2018) Population structure in genetic studies: confounding factors and mixed models. PLoS Genet 14:e1007309. https://doi. org/10.1371/journal.pgen.1007309

Supek F, Bošnjak M, Škunca N, Šmuc T (2011) REVGO summarizes and visualizes long lists of gene ontology terms. PLoS One 6:e21800. https:/doi.org/10.1371/joumal.pone.0021800

Takahashi D, Gorka M, Erban A et al (2019) Both cold and sub-zero acclimation induce cell wall modification and changes in the extracellular proteome in Arabidopsis thaliana. Sci Rep 9. https://doi.org/10.1038/s41598-019-38688-3

Wang D, Liu J, Li C et al (2016) Genome-wide association mapping of cold tolerance genes at the seedling stage in rice. Rice 9:1-10. https://doi.org/10. $1186 / s 12284-016-0133-2$

Wang H, Lee AR, Park SY et al (2018) Genome-wide association study reveals candidate genes related to low temperature tolerance in rice (Oryza sativa) during germination. 3 Biotech 8:235. https://doi.org/10.1007/s13205-018-1252-9

\section{Publisher's Note}

Springer Nature remains neutral with regard to jurisdictional claims in published maps and institutional affiliations. 